\title{
Presynaptic Inhibitory Effects of Acetylcholine in the Hippocampus: A 40-Year Evolution of a Serendipitous Finding
}

\author{
${ }^{\circledR}$ Rita J. Valentino ${ }^{1}$ and ${ }^{\circledR}$ Raymond J. Dingledine ${ }^{2}$ \\ ${ }^{1}$ National Institute on Drug Abuse, Bethesda, Maryland 20892, and ${ }^{2}$ Department of Pharmacology and Chemical Biology, Emory University, \\ Atlanta, Georgia 30322
}

Cholinergic regulation of hippocampal circuit activity has been an active area of neurophysiological research for decades. The prominent cholinergic innervation of intrinsic hippocampal circuitry, potent effects of cholinomimetic drugs, and behavioral responses to cholinergic modulation of hippocampal circuitry have driven investigators to discover diverse cellular actions of acetylcholine in distinct sites within hippocampal circuitry. Further research has illuminated how these actions organize circuit activity to optimize encoding of new information, promote consolidation, and coordinate this with recall of prior memories. The development of the hippocampal slice preparation was a major advance that accelerated knowledge of how hippocampal circuits functioned and how acetylcholine modulated these circuits. Using this preparation in the early 1980s, we made a serendipitous finding of a novel presynaptic inhibitory effect of acetylcholine on Schaffer collaterals, the projections from CA3 pyramidal neurons to dendrites of CA1 pyramidal cells. We characterized this effect at cellular and pharmacological levels, published the findings in the first volume of the Journal of Neuroscience, and proceeded to pursue other scientific directions. We were surprised and thrilled to see that, nearly 40 years later, the paper is still being cited and downloaded because the data became an integral piece of the foundation of the science of cholinergic regulation of hippocampal function in learning and memory. This Progressions article is a story of how single laboratory findings evolve through time to be confirmed, challenged, and reinterpreted by other laboratories to eventually become part of the basis of fundamental concepts related to important brain functions.

\section{Introduction}

The development of in vitro brain slice preparations beginning in the late 1950s and flowering in the 1980s revolutionized neuroscience at the time by opening a window of access to brain neurons through which their biophysical properties, local circuitry, synaptic activity, neurochemistry, and intracellular signaling could be studied without the confounds of anesthetics. The pioneering studies of McIlwain used cortical slices for neurochemical studies (C. L. Li and McIlwain, 1957). The hippocampal slice preparation made its debut in the early 1970s and became a favorite of electrophysiologists because of several advantageous features (Skrede and Westgaard, 1971). The slice maintains its visibly laminar structure of pyramidal and dentate granule neurons, and its accessible afferent and efferent connections and inhibitory interneurons. It was a favorite for those studying seizures and, given the role of hippocampus in memory and learning, it was a staple of plasticity studies.

\footnotetext{
Received Dec. 31, 2020; revised Mar. 26, 2021; accepted Apr. 5, 2021.

The findings and conclusions of this paper are those of the authors and do not necessarily reflect the views of the National Institute on Drug Abuse of the National Institutes of Health, or the U.S. Department of Health and Human Services.

The authors declare no competing financial interests.

Correspondence should be addressed to Rita J. Valentino at valentinorj@nida.nih.gov.

https://doi.org/10.1523/JNEUROSCI.3229-20.2021

Copyright $\odot 2021$ the authors
}

As the late 1970s ushered in the era of the endogenous opioid peptide systems, the hippocampal slice became a favorite of those interested in studying opioid pharmacology and physiology (Deadwyler and Robinson, 1979; Dunwiddie et al., 1980; Lynch et al., 1981). Opioid peptides and their receptors were discovered to be abundant in the hippocampus, making the slice a useful preparation for the study of the acute and chronic pharmacological actions of opioids at molecular, cellular, and circuit levels (Hughes et al., 1977; Duka et al., 1981; Stengaard-Pedersen et al., 1981). In 1980, Ray Dingledine and I joined the ranks of many opioid pharmacologists who traded in their guinea pig ilea and mouse vas deferens for the hippocampal slice. This Progressions article is a classic story of how a set of experimental findings in one laboratory move through time and generations of scientists to be confirmed, challenged, reinterpreted, made more complex, and examined at different levels of analysis to evolve into integral parts of fundamental concepts related to important brain functions. We are thrilled to be a part of that scientific experience.

Serendipity in the absence of a hypothesis

I began a postdoctoral fellowship in the laboratory of Ray Dingledine at the University of North Carolina shortly after he returned from Per Andersen's laboratory in Norway, where the hippocampal slice was developed and being characterized (Skrede and Westgaard, 1971). Ray and I shared a goal of 
characterizing the acute and long-term actions of opioids on hippocampal pyramidal neurons. However, to first test my mastery of electrophysiological approaches in this preparation, I attempted to reproduce the expected and well-characterized excitatory effect of acetylcholine (ACh) on pyramidal neurons that Ray had helped characterize as a postdoctoral fellow (Dodd et al., 1981). It was in that spirit, rather than to test a well-premised hypothesis, that the initial experiments were performed. These led to unexpected findings of cholinergic presynaptic inhibition that were subsequently followed up in a rigorous manner and resulted in the manuscript that appeared in the first volume of the Journal of Neuroscience (Valentino and Dingledine, 1981).

Because the hippocampus was a well-established target of prominent cholinergic projections from the medial septal nucleus and diagonal band of Broca (MS/DBB) with putative functions in arousal, learning, memory, and epilepsy, there were numerous in vivo studies of $\mathrm{ACh}$ actions on hippocampal pyramidal cells (for review, see Hasselmo, 2006). With the advent of the slice preparation, these actions began to be investigated in vitro. Nearly all of these studies provided evidence for an excitatory effect of ACh as characterized by an increase in discharge rate and slow depolarization because of a decreased $\mathrm{K}^{+}$conductance (Dodd et al., 1981; Benardo and Prince, 1982; Cole and Nicoll, 1983). Given those findings, the initial observations of our study, that $\mathrm{ACh}$ iontophoresis produced a rapid decrease of the field EPSP and population spike recorded in CA1 pyramidal neurons evoked by stimulation of Schaffer collaterals, the axons of CA3 pyramidal cells that synapse with CA1 dendrites in the stratum radiatum, was surprising. It was also frustrating for me as a postdoctoral fellow trying to reproduce a well-established excitatory effect. Mapping the response by moving the iontophoretic pipette parallel to the dendritic axis demonstrated that ACh was most potent when applied near the zone of activated synapses and that the reason we did not observe the previously reported slow depolarization was related to my positioning the iontophoretic pipette into the stratum radiatum rather than the stratum pyramidale (Fig. 1). Indeed, when positioned in the pyramidal cell layer, the ACh-induced depolarization reported in previous studies was confirmed.

The decrease in the EPSP produced by ACh application in the stratum radiatum occurred in the absence of a change in the presynaptic action potential, indicating that it was not the result of decreased axonal stimulation. Intracellular recordings revealed a decrease in the magnitude of the EPSP with no change in input resistance or membrane potential, indicating that the effect was synaptic as opposed to a direct effect on CA1 neurons. Together, the results suggested that ACh was acting through presynaptic inhibition. An alternative explanation was that $\mathrm{ACh}$ increased conductance through ion channels in distal dendrites that could not be detected at the soma but could shunt the EPSP current. However, if this were the case, ACh would be expected to alter the shape of the intracellularly recorded EPSP, and it did not. ACh also did not alter the postsynaptic response to glutamate, arguing against this alternative explanation and favoring a mechanism of presynaptic inhibition.

The effect of ACh was substantially reduced by atropine, but not hexamethonium, characterizing it as mediated by muscarinic receptors. Importantly, iontophoretic pulses of ACh localized to the stratum pyramidale also decreased the magnitude of the GABA-mediated recurrent IPSC, indicating that the presynaptic inhibition was not specifically limited to excitatory afferents. Together, the results extended previous findings by Yamamoto

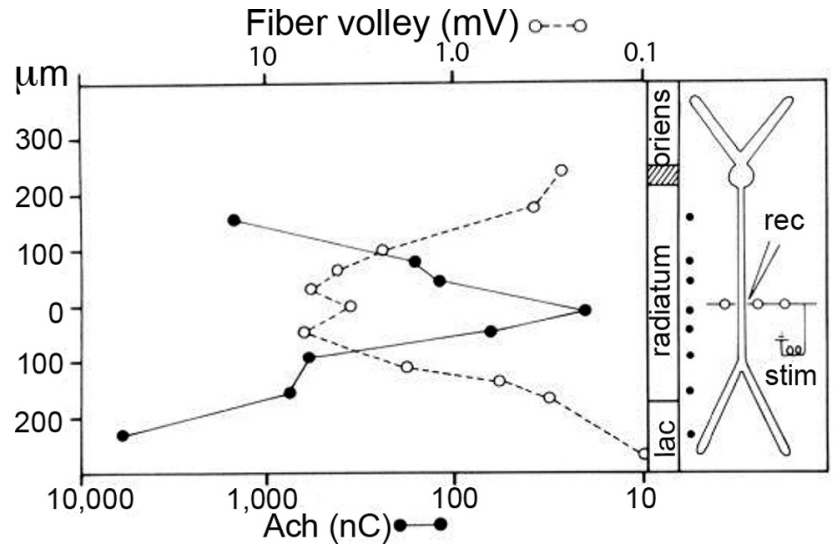

Figure 1. Similar distribution of ACh-sensitive sites and activated excitatory synapses in the CA1 region. Schematic diagram of a CA1 pyramidal cell in the right panel indicates the position of the stimulating (stim) and recording (rec) electrodes in the stratum radiatum. Small black circles in the schematic represent the eight sites in which ACh was iontophoresed. The distance of each site from the recording electrode is indicated in micrometers on the left ordinate. Solid circles in the graph refer to the bottom abscissa and represent the ACh dose, in nanocoulombs (nC, log scale), necessary to reduce a control EPSP by $30 \%$ at each application site. Open circles in the graph refer to the top abscissa and represent the size of the afferent fiber volley (mV, log scale) as the recording electrode was moved in a line parallel to the pyramidal cell axis. lac, Stratum lacunosum moleculare.

and Kawai (1967) in the guinea pig dentate gyrus and of Hounsgaard (1978) in the rat CA1.

The results of the manuscript raised multiple questions, including the mechanism of presynaptic inhibition, whether the observed pharmacological actions were physiologically relevant, when they were engaged, and what was the function of this inhibition with relation to cognitive processes or disease states. These were not questions that Ray and I set about to address, and we returned to our original goals of characterizing opioid effects in the hippocampus. However, with the development of new tools and technologies, new theories evolved that have brought us closer to understanding the functions of $\mathrm{ACh}$ in the hippocampus and particularly the role of cholinergic presynaptic inhibition. These advances included the characterization of multiple ACh receptor subtypes and pharmacological and genetic tools to manipulate them and probe their function. Additionally, in vivo electrophysiological approaches have evolved to be better able to link neural activity to behavior.

\section{Confirming findings and refining interpretations}

Since the publication of our article, there have been several confirmations of the presynaptic inhibitory effects of ACh on Schaffer collaterals (Dutar and Nicoll, 1988; Sheridan and Sutor, 1990; Hasselmo and Schnell, 1994) and on GABA interneurons (Behrends and ten Bruggencate, 1993). The effects observed in hippocampal slices were reproduced in vivo using dialysis administration of $\mathrm{ACh}$ or a cholinesterase inhibitor, and the opposing effect (an increase in the size of the population spike) was produced by the muscarinic antagonist atropine (Herreras et al., 1988b). The effects of the cholinesterase inhibitor and atropine provided evidence for physiological relevance of the effect. Notably, atropine had no effect in the slice preparation in our hands, suggesting that ongoing cholinergic drive from the MS/ DBB was required for this effect (Valentino and Dingledine, 1981). Additional confirmation of our finding came from studies demonstrating that, in the presence of a muscarinic agonist, minimal stimulation of Schaffer collaterals had a higher failure rate 
for evoking EPSCs with no change in EPSC size and also increased paired-pulse ratio, indicative of presynaptic inhibition (Fernandez de Sevilla et al., 2002).

The development of fura-2 to measure calcium influx allowed investigators to combine these measurements with electrophysiological recordings to provide evidence that the muscarinic receptor-mediated inhibition of field EPSPs evoked by stimulation of the stratum radiatum resulted from inhibition of presynaptic voltage-dependent calcium channels, similar to the effect of adenosine (Qian and Saggau, 1997).

Although presynaptic inhibition was observed by us in other hippocampal layers, including the stratum oriens and stratum moleculare of the dentate gyrus, we did not explore these effects in detail. Subsequent studies by other groups showed a greater cholinergic inhibition of Schaffer collateral stimulation in stratum radiatum compared with stimulation of perforant path afferents from entorhinal cortex that synapse in the stratum lacunosum/moleculare or stimulation of the temporoammonic pathway projections from the entorhinal cortex that terminate in CA1 (Hasselmo and Schnell, 1994; Thorn et al., 2017). These differences underscore that the predominant impact of cholinergic inputs will vary across hippocampal subfields.

Notably, cholinergic presynaptic inhibition is not exclusive to the hippocampus. Evidence for it has been demonstrated in intrathalamic, thalamocortical, and intracortical pathways (Hasselmo and Bower, 1992; Hasselmo and Cekic, 1996; Gil et al., 1997; Hsieh et al., 2000; Murakoshi et al., 2001; Blundon et al., 2011; Park et al., 2017) as well as on nociceptive afferents to spinal dorsal horn (D. P. Li et al., 2002), suggesting that this might be a common synaptic motif.

\section{Physiologic relevance}

Because our study and many of the initial studies describing muscarinic presynaptic inhibition were pharmacologic, involving iontophoresis or bath perfusion of hippocampal slices, an important question was whether the effects are physiologically relevant. Early in vivo studies supporting this demonstrated that EPSPs elicited by Schaffer collateral stimulation were decreased during hippocampal theta rhythms induced by sensory stimulation (Herreras et al., 1988a,b) and driven by cholinergic projections from MS/DBB to the hippocampus. Importantly, the attenuation of EPSP magnitude was prevented by atropine and unaffected by GABA antagonists, suggesting that it resulted from presynaptic muscarinic receptor-mediated inhibition. Notably, recurrent inhibition was also reduced, consistent with our finding of AChmediated reductions in recurrent IPSPs. At the same time, potentials recorded in the dentate gyrus evoked by perforant path stimulation were enhanced (Herreras et al., 1988b). This suggested a mechanism by which the septal cholinergic system gates information flow through hippocampal circuits.

MS/DBB cholinergic afferents enter the hippocampus through the stratum oriens/alveus to terminate in CA1, CA3, and the dentate gyrus. Electrical stimulation of this pathway in the slice preparation mimicked the reported inhibitory effects of ACh iontophoresis on field EPSPs evoked by Schaffer collaterals, and this was associated with paired-pulse facilitation, consistent with presynaptic inhibition (Fernandez de Sevilla and Buno, 2003; Buno et al., 2006). Like the effects of ACh application, these effects of electrical stimulation were sensitive to muscarinic, but not nicotinic, receptor antagonists (Fernandez de Sevilla and Buno, 2003).

The optogenetic revolution has generated tools to manipulate circuits with precision and neurochemical selectivity, which is refining our views of circuit function. Optogenetic stimulation of cholinergic MS/DBB terminals decreased the pairedpulse ratio evoked by electrical stimulation in the stratum radiatum and increased that evoked by stimulation in the stratum lacunosum/moleculare, and both effects were mediated by muscarinic receptors: M2 and M3 receptors, respectively (Goswamee and McQuiston, 2019). Because the paired-pulse ratio is thought to be inversely related to presynaptic transmitter release, the interpretation of these results was that the MS/DBB cholinergic input directly inhibited presynaptic transmission in the stratum lacunosum/moleculare, but optogenetic stimulation of the same input indirectly inhibited transmission in the stratum radiatum through GABA interneurons acting on GABAB receptors and opening of GIRK channels on CA1 pyramidal cells. Although the results of this optogenetic study seem at odds with other evidence for cholinergic presynaptic inhibition of Schaffer collaterals, several caveats should be considered. First, the finding that there was no change in conductance in CA1 pyramidal neurons associated with the effect on stratum radiatum inputs, which would be expected with the GABAB-mediated effect, argues against this mechanism. Moreover, an important consideration in studies involving $\mathrm{MS} / \mathrm{DBB}$ stimulation is that $\mathrm{ACh}$ and GABA act as cotransmitters in this pathway that are contained in different vesicles. Their release is regulated by different voltage-dependent calcium channels, and they can auto-regulate and cross-regulate their presynaptic release (Takács et al., 2018). Together, the findings highlight the importance of recognizing caveats of all approaches in interpreting results and also the complexity of interactions of cholinergic terminals within the hippocampus that can contribute to network activity.

Although MS/DBB stimulation invariably alters hippocampal activity, whether ACh acts synaptically or nonsynaptically through volume transmission has been a topic of debate. Because early methods were not sufficiently sensitive to reveal cholinergic synapses, the conventional thinking was that $\mathrm{ACh}$ is released nonsynaptically and acts by "volume transmission." This model was recently challenged in studies using superresolution imaging and electron tomography that demonstrated that all cholinergic terminals form GABAergic synapses and that ACh vesicles dock at synapses (Takács et al., 2018). Although most of the targets of these synapses were dendritic, some could not be classified and might have been terminals. Additionally, spillover of ACh from large vesicles might interact with receptors on terminals.

\section{Pharmacological characterization}

Advances in pharmacology, including the discovery of multiple muscarinic receptor subtypes and their intracellular signaling pathways, the development of pharmacological tools to alter receptor function and the generation of animal models in which these receptors are genetically modified were critical to uncovering the complex effects of ACh in the hippocampus (for review, see Drever et al., 2011). Importantly, these advances provide guidance for developing therapies to treat dysfunctions that have a basis in hippocampal network activity.

Although at the time of our study we were able to distinguish muscarinic from nicotinic receptor effects, the multiplicity of muscarinic receptors subtypes had yet to be discovered. Multiple muscarinic receptors linked to different signaling 
pathways have since been described in the hippocampus in different locations and with different functions (for review, see Drever et al., 2011; Dannenberg et al., 2017). Studies using immunohistochemistry combined with lesions of afferents or immunoelectron microscopy have provided evidence for the localization of M1 and M3 receptors on principal neurons, M2 receptors on interneurons and both cholinergic and noncholinergic presynaptic terminals, and M4 on interneurons and noncholinergic presynaptic terminals (Levey et al., 1995; Rouse et al., 1998, 2000; Yamasaki et al., 2010). The diversity in muscarinic receptor subtypes, their signaling, and cellular and circuit localization add layers of intricacy to cholinergic regulation of hippocampal activity that far exceeded our initial analysis.

Studies using mice lacking different muscarinic receptor subtypes implicated M4 in the suppressant effects of carbachol on EPSPs evoked by Schaffer collateral stimulation (Dasari and Gulledge, 2011). Consistent with this, selective M4-positive allosteric modulators enhanced the suppression of excitatory transmission by muscarinic agonists (Shirey et al., 2008; Thorn et al., 2017). Interestingly, this did not generalize to cholinergic suppression of inhibitory transmission because IPSCs evoked in CA1 pyramidal cells by stimulation of Schaffer collaterals were unaffected by selective M4-positive allosteric modulators and were unchanged in hippocampal slices from mice lacking M4 receptors (Shirey et al., 2008).

Muscarinic presynaptic inhibition of inhibitory transmission is complex and can involve direct activation of M2 receptors on inhibitory terminals or M1/M3-mediated release of endocannabinoids from postsynaptic cells that interact with presynaptic $\mathrm{CB} 1$ receptors to inhibit GABA release (Fukudome et al., 2004). In contrast, a role for endocannabinoids in cholinergic presynaptic inhibition of Schaffer collateral glutamatergic terminals is unlikely because the CB1 receptor is expressed predominantly on inhibitory terminals forming symmetric synapses compared with excitatory terminals forming asymmetric synapses. Moreover, M4 receptors, which are thought to mediate cholinergic presynaptic inhibition, are located presynaptically (Bonilla-Del Río et al., 2019, 2020).

The functional evidence for cholinergic presynaptic inhibition implies that muscarinic receptors should be localized to presynaptic terminals of Schaffer collaterals that are apposed to cholinergic terminals. Indeed, there is evidence for apposition of cholinergic terminals and noncholinergic terminals in the stratum radiatum (Umbriaco et al., 1995). Taken with anatomic evidence for the localization of M2 and M4 muscarinic receptors in presynaptic terminals in CA1, this provides an anatomic substrate for cholinergic presynaptic inhibition.

\section{A place in an evolving conceptual framework}

$\mathrm{MS} / \mathrm{DBB}$ cholinergic projections to the hippocampus regulate network activity that facilitates attention, learning, memory, and sleep-wake transitions. Theta oscillations $(6-10 \mathrm{~Hz})$ induced by activation of this input are critical to these functions. These can be reproduced in vitro in hippocampal slices by perfusing with carbachol in the same doses that produce presynaptic inhibition (Huerta and Lisman, 1993; Natsume and Kometani, 1997; Hyman et al., 2003). Following carbachol washout, pathways that were stimulated during the peak of theta exhibit potentiation. These and other findings demonstrating robust and enduring increases in EPSP amplitude elicited by stimulation of pathways that were in phase with the peak of theta (Huerta and Lisman,
1993; Natsume and Kometani, 1997) have led to the hypothesis that the synchronization of different inputs with phases of theta underlies the encoding of new information and memory formation (Hasselmo et al., 2002). Given that ACh is a primary component of the pathway that generates theta and that it has actions at several sites in the hippocampus, it is of interest to elucidate the specific role of our reported presynaptic inhibition of Schaffer collateral input in these functions.

Hippocampal circuits must be temporally coordinated to encode new information, consolidate, and retrieve information, and these processes need to be distinct and not interfere with each other. To do this, CA1 pyramidal neurons coordinate input from the entorhinal cortex, which relays ongoing information from the environment, with input from CA3, carried by the Schaffer collaterals, which supports retrieval (Hasselmo et al., 2002). Hippocampal theta oscillations driven by cholinergic input from the MS/DBB promote encoding of new information (Hasselmo et al., 2002). In contrast, when ACh levels are low, as in sleep, or when effects are blocked with muscarinic antagonists, consolidation is favored (Dannenberg et al., 2017). The role of cholinergic presynaptic inhibition of Schaffer collateral input may be to bias CA1 activity toward the drive from the entorhinal cortex to favor encoding of new information over retrieval of previous memories that would interfere with that process. In the absence of the cholinergic presynaptic inhibition of Schaffer collateral input, encoding would be impaired, and retrieval favored. The findings that muscarinic receptor antagonists affect retrieval whereas cholinesterase inhibitors enhance encoding of new information would fit this model (Rogers and Kesner, 2003).

While writing this manuscript, the potential relevance of the science for substance use disorders, my current scientific focus, became readily apparent to me. Elucidating how hippocampal circuits that facilitate the formation of new associations are coordinated with those that underlie retrieval of previous associations may be critical to understanding and treating substance use disorders. A primary obstacle to recovery is the driving of drug-taking behaviors by drug-associated cues. By tapping into mechanisms that inhibit retrieval of drug-reward associations while promoting extinction learning and the formation of new associations of nondrug stimuli with reward, the process of recovery may be enhanced and accelerated. Future research on the cholinergic modulation of these hippocampal circuits and new tools to precisely manipulate cholinergic modulation may prove this to be a target to facilitate recovery.

\section{Epilogue}

This study was not part of a master research plan, and neither author followed up on the original findings in a systematic way. That makes it both surprising and rewarding to look back over nearly 40 years to see how the findings eventually found their way fitting into an evolving picture of how the hippocampus functions to regulate learning and memory. Notably, the peak of citations for our manuscript occurred 10 years after publication (1991-1995), and it continues to be cited in 2020. Downloads of the manuscript spiked to $>100 / y r$ in $2013-2020$, nearly 30 40 years after publication. This was my first paper as an electrophysiologist and as a postdoctoral fellow and one of Ray's initial papers as a junior faculty member. We celebrated by smoking cigars in the laboratory, which one could do at the time. It was a 
lasting memory that we both hope to be able to continue to retrieve.

\section{References}

Behrends JC, ten Bruggencate G (1993) Cholinergic modulation of synaptic inhibition in the guinea pig hippocampus in vitro: excitation of GABAergic interneurons and inhibition of GABA-release. J Neurophysiol 69:626-629.

Benardo LS, Prince DA (1982) Cholinergic excitation of mammalian hippocampal pyramidal cells. Brain Res 249:315-331.

Blundon JA, Bayazitov IT, Zakharenko SS (2011) Presynaptic gating of postsynaptically expressed plasticity at mature thalamocortical synapses. J Neurosci 31:16012-16025.

Bonilla-Del Río I, Puente N, Peñasco S, Rico I, Gutiérrez-Rodríguez A, Elezgarai I, Ramos A, Reguero L, Gerrikagoitia I, Christie BR, Nahirney P, Grandes P (2019) Adolescent ethanol intake alters cannabinoid type-1 receptor localization in astrocytes of the adult mouse hippocampus. Addict Biol 24:182-192.

Bonilla-Del Rio I, Puente N, Mimenza A, Ramos A, Serrano M, Lekunberri L, Gerrikagoitia I, Christie BR, Nahirney PC, Grandes P (2020) Acute Delta9-tetrahydrocannabinol prompts rapid changes in cannabinoid CB1 receptor immunolabeling and subcellular structure in CA1 hippocampus of young adult male mice. J Comp Neurol. Advance online publication. Retrieved Dec 23, 2020. doi: 10.1002/cne.25098.

Buno W, Cabezas C, Fernandez de Sevilla D (2006) Presynaptic muscarinic control of glutamatergic synaptic transmission. J Mol Neurosci 30:161164.

Cole AE, Nicoll RA (1983) Acetylcholine mediates a slow synaptic potential in hippocampal pyramidal cells. Science 221:1299-1301.

Dannenberg H, Young K, Hasselmo M (2017) Modulation of hippocampal circuits by muscarinic and nicotinic receptors. Front Neural Circuits 11:102.

Dasari S, Gulledge AT (2011) M1 and M4 receptors modulate hippocampal pyramidal neurons. J Neurophysiol 105:779-792.

Deadwyler SA, Robinson JH (1979) Effects of morphine on hippocampal cells recorded in vitro. Brain Res Bull 4:609-613.

Dodd J, Dingledine R, Kelly JS (1981) The excitatory action of acetylcholine on hippocampal neurones of the guinea pig and rat maintained in vitro. Brain Res 207:109-127.

Drever BD, Riedel G, Platt B (2011) The cholinergic system and hippocampal plasticity. Behav Brain Res 221:505-514.

Duka T, Wuster M, Schubert P, Stoiber R, Herz A (1981) Selective localization of different types of opiate receptors in hippocampus as revealed by in vitro autoradiography. Brain Res 205:181-186.

Dunwiddie T, Mueller A, Palmer M, Stewart J, Hoffer B (1980) Electrophysiological interactions of enkephalins with neuronal circuitry in the rat hippocampus: I. Effects on pyramidal cell activity. Brain Res 184:311-330.

Dutar P, Nicoll RA (1988) Classification of muscarinic responses in hippocampus in terms of receptor subtypes and second-messenger systems: electrophysiological studies in vitro. J Neurosci 8:4214-4224.

Fernandez de Sevilla D, Buno W (2003) Presynaptic inhibition of Schaffer collateral synapses by stimulation of hippocampal cholinergic afferent fibres. Eur J Neurosci 17:555-558

Fernandez de Sevilla D, Cabezas C, Prada AN, Sánchez-Jiménez A, Buño W (2002) Selective muscarinic regulation of functional glutamatergic Schaffer collateral synapses in rat CA1 pyramidal neurons. J Physiol 545:51-63.

Fukudome Y, Ohno-Shosaku T, Matsui M, Omori Y, Fukaya M, Tsubokawa H, Taketo MM, Watanabe M, Manabe T, Kano M (2004) Two distinct classes of muscarinic action on hippocampal inhibitory synapses: $\mathrm{m} 2$ mediated direct suppression and M1/M3-mediated indirect suppression through endocannabinoid signalling. Eur J Neurosci 19:2682-2692.

Gil Z, Connors BW, Amitai Y (1997) Differential regulation of neocortical synapses by neuromodulators and activity. Neuron 19:679-686.

Goswamee P, McQuiston AR (2019) Acetylcholine release inhibits distinct excitatory inputs onto hippocampal CA1 pyramidal neurons via different cellular and network mechanisms. Front Cell Neurosci 13:267.

Hasselmo ME (2006) The role of acetylcholine in learning and memory. Curr Opin Neurobiol 16:710-715.
Hasselmo ME, Bower JM (1992) Cholinergic suppression specific to intrinsic not afferent fiber synapses in rat piriform (olfactory) cortex. J Neurophysiol 67:1222-1229.

Hasselmo ME, Cekic M (1996) Suppression of synaptic transmission may allow combination of associative feedback and self-organizing feedforward connections in the neocortex. Behav Brain Res 79:153-161.

Hasselmo ME, Schnell E (1994) Laminar selectivity of the cholinergic suppression of synaptic transmission in rat hippocampal region CA1: computational modeling and brain slice physiology. J Neurosci 14:3898-3914.

Hasselmo ME, Bodelon C, Wyble BP (2002) A proposed function for hippocampal theta rhythm: separate phases of encoding and retrieval enhance reversal of prior learning. Neural Comput 14:793-817.

Herreras O, Solís JM, Herranz AS, del Río RM, Lerma J (1988a) Sensory modulation of hippocampal transmission: II. Evidence for a cholinergic locus of inhibition in the Schaffer-CA1 synapse. Brain Res 461:303-313.

Herreras O, Solís JM, Muñoz MD, Martín del Río R, Lerma J (1988b) Sensory modulation of hippocampal transmission: I. Opposite effects on CA1 and dentate gyrus synapsis. Brain Res 461:290-302.

Hounsgaard J (1978) Presynaptic inhibitory action of acetylcholine in area CA1 of the hippocampus. Exp Neurol 62:787-797.

Hsieh CY, Cruikshank SJ, Metherate R (2000) Differential modulation of auditory thalamocortical and intracortical synaptic transmission by cholinergic agonist. Brain Res 880:51-64.

Huerta PT, Lisman JE (1993) Heightened synaptic plasticity of hippocampal CA1 neurons during a cholinergically induced rhythmic state. Nature 364:723-725.

Hughes J, Kosterlitz HW, Smith TW (1977) The distribution of methionineenkephalin and leucine-enkephalin in the brain and peripheral tissues. $\mathrm{Br}$ J Pharmacol 61:639-647.

Hyman JM, Wyble BP, Goyal V, Rossi CA, Hasselmo ME (2003) Stimulation in hippocampal region CA1 in behaving rats yields long-term potentiation when delivered to the peak of theta and long-term depression when delivered to the trough. J Neurosci 23:11725-11731.

Levey AI, Edmunds SM, Koliatsos V, Wiley RG, Heilman CJ (1995) Expression of m1-m4 muscarinic acetylcholine receptor proteins in rat hippocampus and regulation by cholinergic innervation. J Neurosci 15:4077-4092.

Li CL, McIlwain H (1957) Maintenance of resting membrane potentials in slices of mammalian cerebral cortex and other tissues in vitro. J Physiol 139:178-190.

Li DP, Chen SR, Pan YZ, Levey AI, Pan HL (2002) Role of presynaptic muscarinic and $\mathrm{GABA}(\mathrm{B})$ receptors in spinal glutamate release and cholinergic analgesia in rats. J Physiol 543:807-818.

Lynch GS, Jensen RA, McGaugh JL, Davila K, Oliver MW (1981) Effects of enkephalin, morphine, and naloxone on the electrical activity of the in vitro hippocampal slice preparation. Exp Neurol 71:527-540.

Murakoshi T, Song SY, Konishi S, Tanabe T (2001) Multiple G-proteincoupled receptors mediate presynaptic inhibition at single excitatory synapses in the rat visual cortex. Neurosci Lett 309:117-120.

Natsume K, Kometani K (1997) Theta-activity-dependent and -independent muscarinic facilitation of long-term potentiation in guinea pig hippocampal slices. Neurosci Res 27:335-341.

Park A, Li Y, Masri R, Keller A (2017) Presynaptic and extrasynaptic regulation of posterior nucleus of thalamus. J Neurophysiol 118:507-519.

Qian J, Saggau P (1997) Presynaptic inhibition of synaptic transmission in the rat hippocampus by activation of muscarinic receptors: involvement of presynaptic calcium influx. Br J Pharmacol 122:511-519.

Rogers JL, Kesner RP (2003) Cholinergic modulation of the hippocampus during encoding and retrieval. Neurobiol Learn Mem 80:332-342.

Rouse ST, Gilmor ML, Levey AI (1998) Differential presynaptic and postsynaptic expression of $\mathrm{m} 1-\mathrm{m} 4$ muscarinic acetylcholine receptors at the perforant pathway/granule cell synapse. Neuroscience 86:221-232.

Rouse ST, Edmunds SM, Yi H, Gilmor ML, Levey AI (2000) Localization of $\mathrm{M}(2)$ muscarinic acetylcholine receptor protein in cholinergic and noncholinergic terminals in rat hippocampus. Neurosci Lett 284:182-186.

Sheridan RD, Sutor B (1990) Presynaptic M1 muscarinic cholinoceptors mediate inhibition of excitatory synaptic transmission in the hippocampus in vitro. Neurosci Lett 108:273-278.

Shirey JK, Xiang Z, Orton D, Brady AE, Johnson KA, Williams R, Ayala JE, Rodriguez AL, Wess J, Weaver D, Niswender CM, Conn PJ (2008) An allosteric potentiator of $\mathrm{M} 4 \mathrm{mAChR}$ modulates hippocampal synaptic transmission. Nat Chem Biol 4:42-50. 
Skrede KK, Westgaard RH (1971) The transverse hippocampal slice: a well-defined cortical structure maintained in vitro. Brain Res 35:589-593.

Stengaard-Pedersen K, Fredens K, Larsson LI (1981) Enkephalin and zinc in the hippocampal mossy fiber System. Brain Res 212:230-233.

Takács VT, Cserép C, Schlingloff D, Pósfai B, Szónyi A, Sos KE, Környei Z, Dénes Á, Gulyás AI, Freund TF, Nyiri G (2018) Co-transmission of acetylcholine and GABA regulates hippocampal states. Nat Commun 9:2848.

Thorn CA, Popiolek M, Stark E, Edgerton JR (2017) Effects of M1 and M4 activation on excitatory synaptic transmission in CA1. Hippocampus 27:794-810.
Umbriaco D, Garcia S, Beaulieu C, Descarries L (1995) Relational features of acetylcholine, noradrenaline, serotonin and GABA axon terminals in the stratum radiatum of adult rat hippocampus (CA1). Hippocampus 5:605620.

Valentino RJ, Dingledine R (1981) Presynaptic inhibitory effect of acetylcholine in the hippocampus. J Neurosci 1:784-792.

Yamamoto C, Kawai N (1967) Presynaptic action of acetylcholine in thin sections from the guinea pig dentate gyrus in vitro. Exp Neurol 19:176-187.

Yamasaki M, Matsui M, Watanabe M (2010) Preferential localization of muscarinic M1 receptor on dendritic shaft and spine of cortical pyramidal cells and its anatomical evidence for volume transmission. J Neurosci 30:4408-4418. 